\title{
Gastric Electrical Stimulation Increases The Proliferation of Interstitial Cells of Cajal and Alters Enteric Nervous System in Diabetic Rats
}

\section{Cai Wang}

Binzhou Medical University Hospital, Binzhou, Shandong, China

Hui Zhang

Binzhou Medical University Hospital, Binzhou, Shandong, China

\section{Le Zhao}

Binzhou Medical University Hospital, Binzhou, Shandong, China

\section{Tao Gao}

Binzhou Medical University Hospital, Binzhou, Shandong, China

\section{Xia Liu}

Binzhou Medical University Hospital, Binzhou, Shandong, China

Yan Chen ( $\square$ chenyanfeihong0906@163.com )

Binzhou Medical University Hospital, Binzhou, Shandong, China

\section{Research Article}

Keywords: gastric electrical stimulation, interstitial cells of cajal, 5-HT2B, enteric nervous system, GDNF

Posted Date: December 2nd, 2020

DOl: https://doi.org/10.21203/rs.3.rs-111658/v1

License: (1) This work is licensed under a Creative Commons Attribution 4.0 International License. Read Full License 
Gastric electrical stimulation increases the proliferation of interstitial cells of cajal and alters enteric nervous system in diabetic rats

Hongcai Wang ${ }^{1 \#}$, Shuhui Zhang ${ }^{2 \#}$, Kaile Zhao ${ }^{2}$, Tao $\mathrm{Gao}^{2}$, Chengxia Liu ${ }^{2 *}$, Yan Chen $^{2 *}$

1. Department of Neurology, Binzhou Medical University Hospital, Binzhou, Shandong, China

2. Department of Gastroenterology, Binzhou Medical University Hospital, Binzhou,

Shandong, China

Running title: GES alters ICC and ENS

Hongcai Wang and Shuhui Zhang are co-first authors.

*Correspondence to:

Yan Chen, $\mathrm{PhD}$,

Department of Gastroenterology,

Binzhou Medical University Hospital,

NO.661 Huanghe $2^{\text {nd }}$ road, Binzhou, Shandong 256603, China.

Tel: $86-0543-3256725$

Fax: 86-0543-3256725

1 E-mail: chenyanfeihong0906@163.com

Chengxia Liu, $\mathrm{PhD}$,

Department of Gastroenterology,

5 Binzhou Medical University Hospital,

6 NO.661 Huanghe $2^{\text {nd }}$ road, Binzhou, Shandong 256603, China.

7 Tel: 86-0543-3256725

8 Fax: 86-0543-3256725

9 E-mail: phdlcx@163.com 
Abstract

Background: Lack of interstitial cells of Cajal (ICC) and neuropathy were the most possible pathological mechanisms of diabetic gastroparesis. Gastric electrical stimulation (GES) is a promising way to treat gastroparesis. The aims of the present study were to explore the impact of GES on ICC together with enteric neurons in diabetic rats and the possible mechanisms involved.

Methods: Sixty rats were randomized into the normal rats, diabetic rats (DM), diabetic rats with sham GES (DM+SGES), and three diabetic rats with GES (DM+GES1, $\mathrm{DM}+\mathrm{GES} 2$ and DM+GES3). The proliferation of ICC and expressions of 5-HT2B, nNOS, CHAT, PGP9.5 and GDNF were evaluated by immunofluorescence staining or Western blot. The expressions of 5-HT in blood and tissue were determined by ELISA.

Results: (1) The proliferation of ICC was hardly observed in the DM group together with the DM+SGES group but increased in the three DM+GES groups. (2) The expression of 5-HT2B was decreased in the DM group and enhanced in the DM+GES groups. Similarly, the expressions of 5-HT in the blood and distal stomach tissue were increased in the DM+GES groups. (3) Both nNOS labeled neurons and CHAT positive neurons were reduced in myenteric plexus of the DM group, while plenty of these neurons were observed the DM+GES groups. (4) The expression of GDNF protein in the diabetic rats was down-regulated, while GES increased the expression of GDNF.

Conclusion: GES improves the proliferation of ICC possibly related with 
5-HT/5-HT2B signal pathway, and alters enteric nervous system partly though the GDNF expression.

Key words: gastric electrical stimulation, interstitial cells of cajal, 5-HT2B, enteric nervous system, GDNF

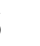

57
prolonged small intestine transit have been recognized as awkward problems in diabetic patients. Delayed gastric emptying, defined as gastroparesis, occurs in $30 \%$ to $50 \%$ of patients with both type 1 and type 2 diabetes [1]. Similarly, prolonged small intestine transit was about one-third of patients with long-standing type 1 diabetes mellitus [2]. Symptoms of dysfunction of upper gastrointestinal motility in diabetes were mainly present as early satiety, abdominal distension, belching, nausea, vomiting and abdominal pain, which seriously affect people's life and work [3]. Because the exact mechanisms of gastrointestinal motility dysfunction remain unclear, the therapeutic effects of traditional prokinetic drugs are far from satisfactory. As it is well-recognized that gastric electric stimulation (GES) with long pulses, namely gastric pacing, could ameliorate gastric emptying and normalize gastric dyshhythmia $[4,5]$, the mechanisms that GES improves the gastric motility need to be further 
illustrated.

Interstitial cells of Cajal (ICC) are extensively distributed in the submucosal, intra-muscular and myenteric plexus layer of the whole gastrointestinal tract. ICC are playing a vital role in gastrointestinal motility because they can generate spontaneously slow wave potential and modulate the neurotransmission from enteric nervous system to smooth muscle cells and regulate the contraction of smooth muscle [6]. He CL et al. showed that the decrease of ICC volume and changes of enteric nerves may be the pathophysiologic mechanism of gastrointestinal complications in a patient with long-standing diabetes [7]. Likewise, loss or damage of ICC also can result in delayed gastric emptying, and abnormal neural transmission in diabetic rodents [8]. Our previous study showed that long-pulse GES improved gastric contraction potentially to promote the proliferation of ICC in diabetic rats [9], but it is not clear how GES promotes ICC proliferation.

Serotonin, also named 5-hydroxytryptamine (5-HT), is a crucial chemical and neurotransmitter mainly distributed in the gastrointestinal tract, blood, and central nervous system. In the whole gut, 5-HT takes effects in gastrointestinal motility, enteric neurogenesis, mucosal maintenance and intestinal inflammation [10]. The 5-HT2B receptor was reported to express in ICC, enteric neurons, and smooth muscle cells [11]. Further, it could mediate the excitatory effect of 5-HT and the immunoreactivity of 5-HT2B receptor was mainly distributed in the longitudinal muscle and myenteric plexus layer of human colon showed by RT-PCR, western blot and immunocytochemistry [12]. Wouters MM et al found 5-HT2B receptors were 
found to be present on ICC and exogenous 5-HT could promote ICC proliferation partly via 5-HT2B receptors in primary cell cultures $[13,14]$. Therefore, the expression of 5-HT2B in diabetic rats and whether GES could affect the 5-HT2B expression need to be investigated.

Diabetic gastroenteropathy has been identified as decrease of ICC, neuropathy, and myopathy of gastrointestinal tract [15]. Alterations of inhibitory and excitatory enteric neurons abundant in the myenteric ganglia were thought to be involved in diabetic neuropathy [16]. The inhibitory enteric neurons (nitrergic neurons mainly expressing nitric oxide synthase) inhibit the motility of gastrointestinal tract, while the excitatory enteric neurons mean the cholinergic neurons principally expressing choline acetyltransferase (ChAT) and accelerate the motility. Deficiency of neuronal nitric oxide synthase (nNOS) was documented in the antral myenteric plexus of diabetic rats $[17,18]$. Previous study illustrated that the degeneration of nNOS in the gastric pylorus of diabetic rats went through loss of number and function of nitrergic nerve fibers and further defect of function in neurons cell bodies of the ganglia [19]. $\mathrm{Du} F$ et al found that enteric neuropathy (both decreased nNOS and CHAT neurons) was noted in the stomach of diabetic rats, and further these changes were considered to be regulated, in part, according to a decreased expression of glia cell line-derived neurotrophic factor (GDNF) [20, 21]. GDNF is a crucial neurotrophic factor for the proliferation, maturation, migration, maintenance and survival of enteric neurons. However, no significant reduction of cholinergic nerve was found in antrum, jejunum, and ileum of diabetic rats $[22,23]$. Thus, the effects of GES on enteric nervous system 
need further to be clarified.

The objectives of this study were to explore the mechanism of GES on proliferation of ICC and 5-HT/5HT-2B pathway was involved and further to illustrate the effects and possible mechanism of GES on enteric nervous system.

\section{Experimental Animals}

Male Sprague-Dawley rats $(6-8$ weeks, $\mathrm{N}=60)$ were purchased from Jinan Pengyue Experimental Animal Breeding Co., Ltd. (Shandong, China) and were allowed to the standard residence with controlled room conditions (temperature for $22 \pm 0.5^{\circ} \mathrm{C}$ and humidity for $55 \pm 10 \%$ with 12 -hour light/dark cycle). All experimental procedures were officially approved by the Animal Care and Use Committee and the animal research was conducted following the ethical guidelines of Laboratory Animal Ethical Committee.

\section{Diabetic Model}

Animal models (50 of 60 rats) were developed diabetes by intraperitoneal injecting streptozotocin (STZ, $60 \mathrm{mg} / \mathrm{kg}$, Sigma-Aldrich, United States). Ten rats injected same volume of solvent were taken as the normal control group. All of the rats were forbidden for drinking in the 4 hours afterwards. One week afterwards, diabetic model was successfully determined by twice blood glucose in the tail vein $>$ $16.7 \mathrm{mmol} / \mathrm{L}$. The mental state, fur, diet, water intake, urine output, and weight of the rats were also observed. 


\section{Electrodes Implantation}

Before diabetic models established, 40 of 50 these diabetic rats were allowed to implant electrodes (A\&E Medical, Farmingdale, NJ) in the stomach for stimulation. After anesthesia, the abdomen was opened and a pair of electrodes was embedded in the serosa layer of the greater curvature, located at $2 \mathrm{~cm}$ above the pylorus. The space of the electrodes was kept at $0.5 \mathrm{~cm}$ apart. The other end of the wires was crossed through the abdominal wall and subcutaneously went through under the skin to the posterior neck. The ends of the wires were linked to an electrical stimulator (YC-2-S, Chengdu instrument factory, China).

\section{Experimental Protocol}

All of the rats were grouped into the normal control group, diabetic group (DM), diabetic with sham GES group (DM+GES), diabetic with GES group (DM+GES). On the basis of different stimulation pulse width [9], the DM+GES group were randomized into three subgroups: DM+GES1 (100 ms), DM+GES2 (300 ms), and DM+GES3 (550 ms). The GES with frequency $5.5 \mathrm{cpm}$ and current $4 \mathrm{~mA}$ was given for 6 weeks with $30 \mathrm{~min} / \mathrm{d}$, while the wires were only connected to the stimulator with no current in the DM+SGEP group. At last, after all rats were sacrificed, blood and gastric antrum tissues were obtained for ELISA, western blot and immunofluorescence staining.

\section{ELISA for 5-HT}

Blood samples were acquired from abdominal aortic vein and centrifuged at $3000 \mathrm{rpm}$ for $15 \mathrm{~min}$. Stomach tissue homogenates $(100 \mathrm{mg}$ tissue in $1 \mathrm{ml}$ of ice-cold 
$1 \times$ PBS) were obtained and centrifuged for 15 minutes at $5000 \mathrm{rpm}$. Supernatant was obtained and kept at $-80^{\circ} \mathrm{C}$. The 5 -HT concentration was tested by an ELISA kit (MBS288208, MyBioSource, USA) following the manufacturer's instruction. Optical density (OD) of samples and standard at $450 \mathrm{~nm}$ was recorded by an ELISA plate scanner.

\section{Immunofluorescence staining}

To analyze the expression of ICC proliferation and 5-HT2B, the stomach tissue was fixed in $4 \%$ paraformaldehyde, made into paraffin blocks, and sliced for $5 \mu \mathrm{m}$. The sections were kept in xylene and deparaffinize for 10 min and gradually hydrated in a series of graded alcohols. Retrieved antigen by a water bath at $92-95^{\circ} \mathrm{C}$ and blocked nonspecific reaction by fetal bovine serum, the antibody of c-kit $(1: 100$, Santa Cruz, USA), ki67 (1:100, Abcam, Cambridge, UK), and 5-HT2B (1:100, Abcam, Cambridge, UK) were added to the sections and incubated at $4^{\circ} \mathrm{C}$ overnight (Table 1). After labeled by secondary antibody for $1 \mathrm{~h}$ at room temperature and stained DAPI for about $5 \mathrm{~min}$, the slices were observed under a confocal laser scanning microscope (Nikon, Japan).

For whole-mount staining of nNOS, CHAT, PGP9.5, stomach tissue was mount on a Sylgard dish and kept in Zamboni's fixative for at least $24 \mathrm{~h}$. Myenteric plexus layer was obtained by sharp dissection. After rinsed with PBS with $0.3 \%$ Triton X-100 for 6 times, the sample was kept in 5\% normal bovine serum for $60 \mathrm{~min}$ to get out of non-specific staining. The primary antibody of nNOS (1:500; Abcam, Cambridge, UK), CHAT (1:100; Abcam, Cambridge, UK), PGP9.5 (1:1000; Abcam, Cambridge, 
UK) was incubated overnight at $4{ }^{\circ} \mathrm{C}$ (Table 1). After secondary antibody for $60 \mathrm{~min}$ and then DAPI for $5 \mathrm{~min}$, the sample was flattened on a slide. Pictures could be taken under the confocal laser scanning microscope.

\section{Western blot analysis}

The stomach tissue was grinded with RIPA buffer and protease inhibitor for 30 min. After centrifugation at $12000 \mathrm{~g}$ at $4^{\circ} \mathrm{C}$ for $10 \mathrm{~min}$, the supernatant was gotten as the total proteins. The protein concentration was determined by BCA reagent (Pierce, Rockford, IL, United States). $10 \%$ sodium dodecyl sulfate-polyacrylamide gel electrophoresis was employed to separate the whole proteins $(20 \mu \mathrm{g})$ according to the molecular weight. Different proteins were completely transferred to PVDF membranes (Millipore, United States). After blocked by 5\% skim milk solution for about $60 \mathrm{~min}$, the membranes were reacted with primary antibodies against GDNF (1:200, Abcam, United Kingdom), GAPDH (1:10000, Abcam, United Kingdom) at $4^{\circ} \mathrm{C}$ overnight. Secondary antibodies were also added to the membranes for $60 \mathrm{~min}$ at room temperature. The bands of different protein on the PVDF membranes were revealed by enhanced chemiluminescence kit (Amersham Pharmacia, United States). Finally, the densitometry of blots was analyzed by Image J software.

\section{Statistical Analysis}

SPSS version 24.0 (SPSS Inc., Chicago, IL) was employed to perform statistical analyses. All of the data were showed as Mean \pm SEM and ANOVA was applied to determine statistical differences among multiple groups. LSD test was applied when the variance was equal, while Dunnett's T3 test was to conduct when equal variance 
was not achieved. $\mathrm{P}$ values less than 0.05 were taken as statistically significant association.

\section{Results}

Effects of GES on the proliferation of ICC

As indicated in Figure 1, plenty of c-kit+ cells with a small number of Ki67+ cells were showed in the area of antrum in the control group. Few c-kit $+\mathrm{Ki67}+$ cells in the DM group could be observed as well as in the DM+SGES group (both $\mathrm{P}<0.001$ vs. control group). However, compared with the DM group, a large amount of c-kit+ cells with an increased number of Ki67+ cells in the intermuscular and muscular layer were found in the DM+GES group (DM+GES1: $\mathrm{P}=0.002 ; \mathrm{DM}+\mathrm{GES} 2: \mathrm{P}=0.001$; $\mathrm{DM}+\mathrm{GES} 3: \mathrm{P}=0.001)$.

\section{Effects of GES on the expression of 5-HT2B}

Figure 2 showed the expression of 5-HT2B in different groups by immunofluorescence and Western blot. In the control group, colocalization of 5-HT2B + and c-kit+ cells were mainly showed in the intermuscular and muscular layer. In the DM group together with the DM+SGES group, there were rare 5-HT2B+/c-kit+ cells in the muscular and intermuscular layer (both $\mathrm{P}=0.002$ vs. control group). In the meantime, three different long pulse GES enhanced the quantity of the 5-HT2B+/c-kit+ cells (DM+GES1: $\mathrm{P}=0.002 ; \mathrm{DM}+\mathrm{GES} 2: \mathrm{P}=0.002 ; \mathrm{DM}+\mathrm{GES} 3$ : $\mathrm{P}=0.004$; vs. DM group).

\section{Effects of GES on the level of 5-HT}



deducted when compared with normal rats $(\mathrm{P}<0.001)$. As expected, there was no statistic difference observed in the DM+SGES group and the DM group $(\mathrm{P}=0.125)$. However, it was comparative between the DM+GES groups (markedly increased) and the DM group (all $\mathrm{P}<0.001)$.

According to Figure 3B, the tissue level of 5-HT in the DM group was also reduced in the same degree ( $\mathrm{P}=0.018$ vs. control group). No significant evidence was 
were detected in the enteric nerve system. Compared the control group, the expression of CHAT staining neurons were changed in the DM group and the DM+SGES group ( $\mathrm{P}=0.001$ and $\mathrm{P}=0.005$ ). Meanwhile, three different long pulse GES improved the expression of CHAT nerves (all $\mathrm{P}<0.001$, vs. the DM group).

\section{Effects of GES on the expression of GDNF protein}

As indicated in Figure 6, the GDNF protein expression in the DM group was dramatically decreased compared with the control group $(\mathrm{P}<0.001)$. It was nearly no significant difference in the diabetic rats with and without SGES $(\mathrm{P}=0.650)$. However, it was suggested that the GDNF protein expressions were obviously enhanced in the diabetic rats with different GES compared with the diabetic rats without GES (all $\mathrm{P}<0.001)$.

\section{Discussion}

In the present study, reduced number of ICC with lack of proliferation was found in the gastric antrum of diabetic rats, and further it is noticeable that the 5-HT/5-HT2B signal pathway was also decreased. Meanwhile, both nitrogen and cholinergic neurons were decreased to some extent with down-regulated GDNF expression. GES increased the quantity of ICC though stimulating the proliferation of ICC partly associated with 5-HT/5-HT2B signal pathway. At the same time, GES also improved the survival of nitrogen and cholinergic neurons, in part, with the GDNF expression.

Interstitial cells of cajal (ICC) are well-known as pace makers to initiate slow 
waves in the gut, which are the promoter of motility. It is reported that depletion or ultrastructural changes of ICC in the stomach were revealed in diabetic patients with delayed gastric emptying [24]. T Ordög et al also showed that in diabetic NOD/LtJ mice, ICC was found to be greatly reduced through immunofluorescence in the distal stomach, resulting in delayed gastric emptying, impaired electrical pacemaking, and reduced motor neurotransmission [8]. We also showed ICC reduced in the diabetic rats, which is coincident with the previous findings. Plasticity of ICC in physiological condition and some disorders is achieved by transdifferentiation and apoptosis or by proliferation, survival of the remaining ICC, and mature from precursor cells [25]. Although the specific mechanisms were still unclear, electric stimulation was verified to be effective with alteration of the transmembrane potential of cells to inhibit apoptosis or promote proliferation [26]. As reported in our previous study, long pulse GES could suppress the ICC apoptosis though TUNEL staining in diabetic rats. In this study, proliferation of ICC was concentrated to identify using Ki67 staining in the gastric wall of diabetic rats with GES treatment.

The possible mechanisms participated in ICC proliferation arouse people's interest. 5-HT binding to 5-HT2B receptor could take part in cell survival and proliferation. The 5-HT2B receptor was reported to express on ICC, and the proliferation of ICC could be triggered by activating the 5-HT2B receptor [14]. In the further study in vitro on primary cell cultures of ICC in mouse jejunum, exogenous 5-HT could regulate the quantity of ICC by promoting the proliferation of ICC depending on 5-HT2B receptors [13]. A study in vivo using transgenic mice found 
that Ki67 labeled proliferating ICC were lower about 30\% in the myenteric layer and $40 \%$ in deep muscular plexus layer in the jejunum of $\mathrm{Htr} 2 \mathrm{~b}(-/-)$ mice, indicating ICC could proliferate in adult mice and the signaling 5-HT2B receptor is crucial for maintenance of ICC networks [27]. Borman RA using electrical field stimulation to intervene the longitudinal muscle samples of human colon showed that the contractile response could be enhanced by adding 5-HT, while selective 5-HT2B receptor antagonists could inhibit the contractile response to 5-HT [28]. In this study, we found the expression of 5-HT2B and plasma and tissue 5-HT were significantly decreased, and different width GES could improve the 5-HT2B expression and 5-HT level, revealing that GES may regulate the 5-HT/5-HT2B receptor pathway to promote the proliferation of ICC.

Till now, the pathogenesis of diabetic gastropathy is not fully understood. The most accepted mechanisms of gastroparesis are defect of ICC and loss of expression of nNOS [29]. Nitric oxide (NO) is synthesized by nNOS and secreted by inhibitory nitrergic neurons to relax the smooth muscle in the gastrointestinal tract. It is documented that NOS expression in the myenteric plexus was significantly decreased in antrum, contributing to diabetic emptying in streptozotocin-induced diabetic rats and gastric relaxation in spontaneously diabetic rats $[17,18]$. Consist with the previous study, we also found the expression of nNOS was reduced in the myenteric plexus according to the whole-mount staining. Otherwise, chronic electrical stimulation in vivo of anterior and extensor digitorum longus could improve the nNOS expression in skeletal muscle to regulate the muscle activity [30]. Hoque KE et 
al reported that electrical stimulation of the hippocampal fimbria could facilitate the nNOS activity in the medial shell of the rat [31]. In the study, although the expression of nNOS was reduced in the myenteric plexus of gastric wall in diabetic rats, GES could increase the expression of nNOS for 6 weeks, remaindering that the alteration of nNOS expression may play a role in the gastric motility.

In another aspect, CHAT in excitatory neurons takes effects on the neurotransmitter acetylcholine. It has reported that an increased density of CHAT-positive nerve fibers in the myenteric layer of guinea pigs jejunum for 5-6 weeks' diabetes contributed to gastrointestinal dysfunctions [32]. However, others showed that the amount of CHAT was reduced in the proximal and distal colon of streptozotocin-induced diabetic rats for 8 weeks, but it was not reduced in the 4 weeks $[20,21]$. In the study, we found that the CHAT neurons in the myenteric layer of gastric antrum were reduced in diabetic rats for more than 6 weeks. The difference of the results was speculated to be related with different animals, location of the gut or disease duration. Moreover, electrical stimulation of the dorsal hippocampus reduced about $40 \%$ of hippocampal ChAT activity in BALB/c mice [33]. However, in the study, we revealed that GES increased the CHAT expression locating in the myenteric neurons of diabetic rats. This may provide ideas of diabetic gastropathy relevant to loss of ICC, nNOS and CHAT neurons.

GDNF took a vital role in the pathogenesis to induce diabetic neuropathy, which could provide nutriment for enteric nervous system. Anitha $\mathrm{M}$ et al reported that GDNF could rescue the enteric neuronal survival from the effects of hyperglycemia in 
primary enteric neurons and reverse the myenteric neuronal apoptosis in streptozotocin-induced diabetic mice [34]. Similarly, GDNF was identified to be reduced in the colon of streptozotocin-induced diabetic rat $[20,21]$. Others also pointed out that attenuation of GDNF was found in 2 weeks' diabetes to result in structural alterations and defective myelination in the stuy of sciatic nerves. Nevertheless, peripheral GDNF gene delivery could up-regulate the GDNF expression in the peripheral nervous system [35]. A study showed that short-term electrical stimulation with low frequency reduced GDNF secretion by the skeletal muscle, while long-term electrical stimulation up-regulated the GDNF production [36]. Other previously study also indicated in rat models that daily electrical muscle stimulation for three-month improved the GDNF expression in muscles to accelerate neural regeneration following nerve injury [37]. Our study also found that GES for 6 weeks could increase the expression of GDNF, meaning that GES possibly improve the GDNF expression to alter the myenteric neurons.

In conclusion, the number of ICC was reduced in the gastric antrum of diabetic rats accompanying with reduced 5-HT2B expression and 5-HT level in plasma and tissue. On the other aspect, nNOS and CHAT neurons in myenteric layer were decreased with downregulated GDNF secretion. Both loss of ICC and neuropathy led to diabetic gastroparesis. Long pulse GES facilitated the number of proliferative ICC probably via upregulated 5-HT/5-HT2B signal pathway. Simultaneously, GES also rescued the survival of nitrogen and cholinergic neurons potentially with the increased GDNF expression. This study provides a theoretical basis for the clinical application 
of GES in the treatment of gastroparesis.

\section{Declaration}

\section{Acknowledgement}

We declare that we do not have any commercial or associate interest in connection with the work submitted.

\section{Author contribution}

Shuhui Zhang, Kaile zhao and Tao Gao performed the experiments. Hongcai Wang and Chengxia Liu gave suggestions in the study and discussed the data. Yan Chen drafted the manuscript and also took responsibility of the data analysis.

\section{Availability of data and material}

The datasets used and/or analyzed during the current study are available from the corresponding author on reasonable request.

\section{Funding}

This work has been supported by the National Natural Science Foundation of China (NO. 81700472) and the Medicine and Health Care Science and Technology Development Plan Projects Foundation of Shandong Province (No. 2016WS0021).

\section{Ethics approval and consent to participate}

All experimental procedures were officially approved by the Animal Care and Use Committee and the animal research was conducted following the ethical guidelines of Laboratory Animal Ethical Committee.

\section{Consent for publication}




\section{Competing interests}

Not applicable.

\section{Reference}

1. Horowitz M, Wishart JM, Jones KL, Hebbard GS. Gastric emptying in diabetes: an overview. Diabet Med. 1996;13:S16-S22.

2. Faria M, Pavin EJ, Parisi MC, et al. Delayed small intestinal transit in patients with long-standing type 1 diabetes mellitus: investigation of the relationships with clinical features, gastric emptying, psychological distress, and nutritional parameters. Diabetes Technol Ther. 2013;15:32-38.

3. Krishnan B, Babu S, Walker J, Walker AB, Pappachan JM. Gastrointestinal complications of diabetes mellitus. World J Diabetes. 2013;4:51-63.

4. McCallum RW, Chen JD, Lin Z, Schirmer BD, Williams RD, Ross RA. Gastric pacing improves emptying and symptoms in patients with gastroparesis. Gastroenterology. 1998;114:456-461.

5. Hocking MP, Vogel SB, Sninsky CA. Human gastric myoelectric activity and gastric emptying following gastric surgery and with pacing. Gastroenterology. 1992;103:1811-1816.

6. Takaki M. Gut pacemaker cells: the interstitial cells of Cajal (ICC). J Smooth Muscle Res. 2003;39:137-161.

7. He CL, Soffer EE, Ferris CD, Walsh RM, Szurszewski JH, Farrugia G. Loss of interstitial cells of cajal and inhibitory innervation in insulin-dependent diabetes. Gastroenterology. 2001;121:427-434.

8. Ordög T, Takayama I, Cheung WK, Ward SM, Sanders KM. Remodeling of networks of interstitial cells of Cajal in a murine model of diabetic gastroparesis. Diabetes. 2000;49:1731-1739.

9. Chen Y, Wang H, Li H, Liu S. Long-Pulse Gastric Electrical Stimulation Repairs Interstitial Cells of Cajal and Smooth Muscle Cells in the Gastric Antrum of 
Diabetic Rats. Gastroenterol Res Pract. 2018;2018:6309157.

10. Gershon MD. 5-Hydroxytryptamine (serotonin) in the gastrointestinal tract. Curr Opin Endocrinol Diabetes Obes. 2013;20:14-21.

11. Wouters MM, Farrugia G, Schemann M. 5-HT receptors on interstitial cells of Cajal, smooth muscle and enteric nerves. Neurogastroenterol Motil. 2007;19 Suppl 2:5-12.

12. Borman RA, Tilford NS, Harmer DW, et al. 5-HT(2B) receptors play a key role in mediating the excitatory effects of 5-HT in human colon in vitro. Br J Pharmacol. 2002;135:1144-1151.

13. Wouters MM, Gibbons SJ, Roeder JL, et al. Exogenous serotonin regulates proliferation of interstitial cells of Cajal in mouse jejunum through 5-HT2B receptors. Gastroenterology. 2007;133:897-906.

14. Wouters MM, Roeder JL, Tharayil VS, et al. Protein kinase C\{gamma\} mediates regulation of proliferation by the serotonin 5-hydroxytryptamine receptor $2 \mathrm{~B}$. $J$ Biol Chem. 2009;284:21177-21184.

15. Yarandi SS, Srinivasan S. Diabetic gastrointestinal motility disorders and the role of enteric nervous system: current status and future directions. Neurogastroenterol Motil. 2014;26:611-624.

16. Chandrasekharan B, Srinivasan S. Diabetes and the enteric nervous system. Neurogastroenterol Motil. 2007;19:951-960.

17. Wrzos HF, Cruz A, Polavarapu R, Shearer D, Ouyang A. Nitric oxide synthase (NOS) expression in the myenteric plexus of streptozotocin-diabetic rats. Dig Dis Sci. 1997;42:2106-2110.

18. Takahashi T, Nakamura K, Itoh H, Sima AA, Owyang C. Impaired expression of nitric oxide synthase in the gastric myenteric plexus of spontaneously diabetic rats. Gastroenterology. 1997;113:1535-1544.

19. Cellek S, Foxwell NA, Moncada S. Two phases of nitrergic neuropathy in streptozotocin-induced diabetic rats. Diabetes. 2003;52:2353-2362.

20. Du F, Liu S. Electroacupuncture with high frequency at acupoint ST-36 induces regeneration of lost enteric neurons in diabetic rats via GDNF and PI3K/AKT 
signal pathway. Am J Physiol Regul Integr Comp Physiol. 2015;309:R109-R118.

21. Du F, Wang L, Qian W, Liu S. Loss of enteric neurons accompanied by decreased expression of GDNF and PI3K/Akt pathway in diabetic rats. Neurogastroenterol Motil. 2009;21:1229-e114.

22. Demedts I, Masaoka T, Kindt S, et al. Gastrointestinal motility changes and myenteric plexus alterations in spontaneously diabetic biobreeding rats. $J$ Neurogastroenterol Motil. 2013;19:161-170.

23. Lincoln J, Bokor JT, Crowe R, Griffith SG, Haven AJ, Burnstock G. Myenteric plexus in streptozotocin-treated rats. Neurochemical and histochemical evidence for diabetic neuropathy in the gut. Gastroenterology. 1984;86:654-661.

24. Bashashati M, McCallum RW. Is Interstitial Cells of Cajal-opathy Present in Gastroparesis?. J Neurogastroenterol Motil. 2015;21:486-493.

25. Al-Shboul OA. The importance of interstitial cells of cajal in the gastrointestinal tract. Saudi J Gastroenterol. 2013;19:3-15.

26. Love MR, Palee S, Chattipakorn SC, Chattipakorn N. Effects of electrical stimulation on cell proliferation and apoptosis. $J$ Cell Physiol. 2018;233:1860-1876.

27. Tharayil VS, Wouters MM, Stanich JE, et al. Lack of serotonin 5-HT2B receptor alters proliferation and network volume of interstitial cells of Cajal in vivo. Neurogastroenterol Motil. 2010;22:462-e110.

28. Borman RA, Tilford NS, Harmer DW, et al. 5-HT(2B) receptors play a key role in mediating the excitatory effects of 5-HT in human colon in vitro. Br J Pharmacol. 2002;135:1144-1151.

29. Oh JH, Pasricha PJ. Recent advances in the pathophysiology and treatment of gastroparesis. J Neurogastroenterol Motil. 2013;19:18-24.

30. Reiser PJ, Kline WO, Vaghy PL. Induction of neuronal type nitric oxide synthase in skeletal muscle by chronic electrical stimulation in vivo. J Appl Physiol (1985). 1997;82:1250-1255.

31. Hoque KE, Blume SR, Sammut S, West AR. Electrical stimulation of the hippocampal fimbria facilitates neuronal nitric oxide synthase activity in the 
medial shell of the rat nucleus accumbens: Modulation by dopamine D1 and D2 receptor activation. Neuropharmacology. 2017;126:151-157.

32. LePard KJ. Choline acetyltransferase and inducible nitric oxide synthase are increased in myenteric plexus of diabetic guinea pig. Auton Neurosci. 2005;118:12-24.

33. Micheau J, Destrade C, Jaffard R. Physostigmine reverses memory deficits produced by pretraining electrical stimulation of the dorsal hippocampus in mice. Behav Brain Res. 1985;15:75-81.

34. Anitha M, Gondha C, Sutliff R, et al. GDNF rescues hyperglycemia-induced diabetic enteric neuropathy through activation of the PI3K/Akt pathway. J Clin Invest. 2006;116:344-356.

35. Liu GS, Shi JY, Lai CL, et al. Peripheral gene transfer of glial cell-derived neurotrophic factor ameliorates neuropathic deficits in diabetic rats. Hum Gene Ther. 2009;20:715-727.

36. Vianney JM, Miller DA, Spitsbergen JM. Effects of acetylcholine and electrical stimulation on glial cell line-derived neurotrophic factor production in skeletal muscle cells. Brain Res. 2014;1588:47-54.

37. Willand MP, Rosa E, Michalski B, et al. Electrical muscle stimulation elevates intramuscular BDNF and GDNF mRNA following peripheral nerve injury and repair in rats. Neuroscience. 2016;334:93-104.

Table 1. List of primary and secondary antibodies.

\begin{tabular}{llll}
\hline Primary antibody & Host & Dilution & Source \\
\hline c-kit & Mouse & $1: 100$ & Santa Cruz \\
Ki67 & Rabit & $1: 100$ & Abcam \\
5-HT2B & Rabit & $1: 100$ & Abcam \\
nNOS & Rabit & $1: 500$ & Abcam \\
CHAT & Rabit & $1: 100$ & Abcam \\
PGP9.5 & Mouse & $1: 1000$ & Abcam \\
GDNF & Mouse & $1: 1000$ & Abcam \\
\hline Secondary antibody & Host & Dilution & Source \\
\hline Mouse IgG & Goat anti-mouse Alexa 488/594 & $1: 1000$ & Invitrogen
\end{tabular}




\begin{tabular}{llll} 
Rabit IgG & Goat anti-rabit Alexa 594/488 & $1: 1000$ & Invitrogen \\
Rabit IgG & Donkey anti-rabit Alexa 594/488 & $1: 1000$ & Invitrogen \\
Mouse IgG & Donkey anti-mouse Alexa 488/594 & $1: 1000$ & Invitrogen \\
\hline
\end{tabular}

Figure 1. Representative images of immunofluorescent staining for proliferated ICC labeled with c-kit (green), Ki67 (red) and DAPI (blue) in different groups. Lots of c-kit+ cells were located in the muscle and myenteric layer with dispersed Ki67+ nucleuses in the control group. An obviously decreased amount of c-kit+ cells and rarely $\mathrm{Ki67}+$ nucleuses were identified in the gastric antrum of the DM and $\mathrm{DM}+\mathrm{SGES}$ group. In the DM+GES groups, numerous c-kit+ cells collocated with Ki67+ nucleuses were found. Quantification of numbers of c-kit/ki67+ cells in different groups. $* \mathrm{P}<0.05$ compared with the $\mathrm{DM}$ group.

Figure 2. Confocal micrographs of 5-HT2B in the gastric antrum of different groups. The collocation of c-kit + cells and 5-HT2B + cells was in the muscle and myenteric layer in the normal rats. In the DM and DM+SGES group, the immune staining of c-kit + and 5-HT2B + cells were markedly reduced. 5-HT2B+/c-kit + cells were observed to distribute in the intramuscular and myenteric layer. Scale bars $=20 \mathrm{~mm}$. Quantification of numbers of 5 -HT2B+/c-kit + cells in different groups. $* \mathrm{P}<0.05$ compared with the DM group.

Figure 3. The plasma and tissue level of 5-HT in each group. Both the plasma and tissue 5-HT level in the DM group were significantly reduced compared the control group. However, the plasma and tissue IGF-1 level in the DM+GES groups. ${ }^{*} \mathrm{P}<0.05$ compared with the DM group.

Figure 4. Confocal micrographs of cholinergic neurons labeled with PGP9.5 (red) and nNOS (green) in the myenteric layer. In the control group, a large amount of PGP9.5+/nNOS + neurons were noted in the ganglia. Visual reductions of PGP9.5+/nNOS+ neurons were present in the DM and DM+SGES group. A remarkably increased PGP9.5+/nNOS+ neurons were showed in the DM+GES groups. 
527 Quantification of numbers of PGP9.5+/nNOS+ neurons in different groups. ${ }^{*} \mathrm{P}<0.05$

528 compared with the DM group.

530 Figure 5. Confocal micrographs of cholinergic neurons labeled with PGP9.5 (green) 531 and CHAT (red) in the myenteric layer. In the control group, plenty of CHAT staining 532 neurons were collocated with PGP9.5 staining neurons, but a few of PGP9.5+/ 533 CHAT + neurons were found in the ganglia of the DM and DM+SGES group. In the 534 DM+GES group, numerous PGP9.5+/CHAT+ neurons were observed in the myenteric layer. Quantification of numbers of PGP9.5+/CHAT+ neurons in different 536 groups. $* \mathrm{P}<0.05$ compared with the $\mathrm{DM}$ group.

538 Figure 6. Expression of GNDF protein in the gastric antrum. Compared with the 539 control group, the expression of GNDF protein was obviously decreased in the DM 540 group. There was no significant change of GNDF protein between the DM and 541 DM+SGES group. The expressions of GNDF protein in the DM+GES groups were improved in the DM+GES groups. ${ }^{*} \mathrm{P}<0.05$ compared with the DM group. 


\section{Figures}

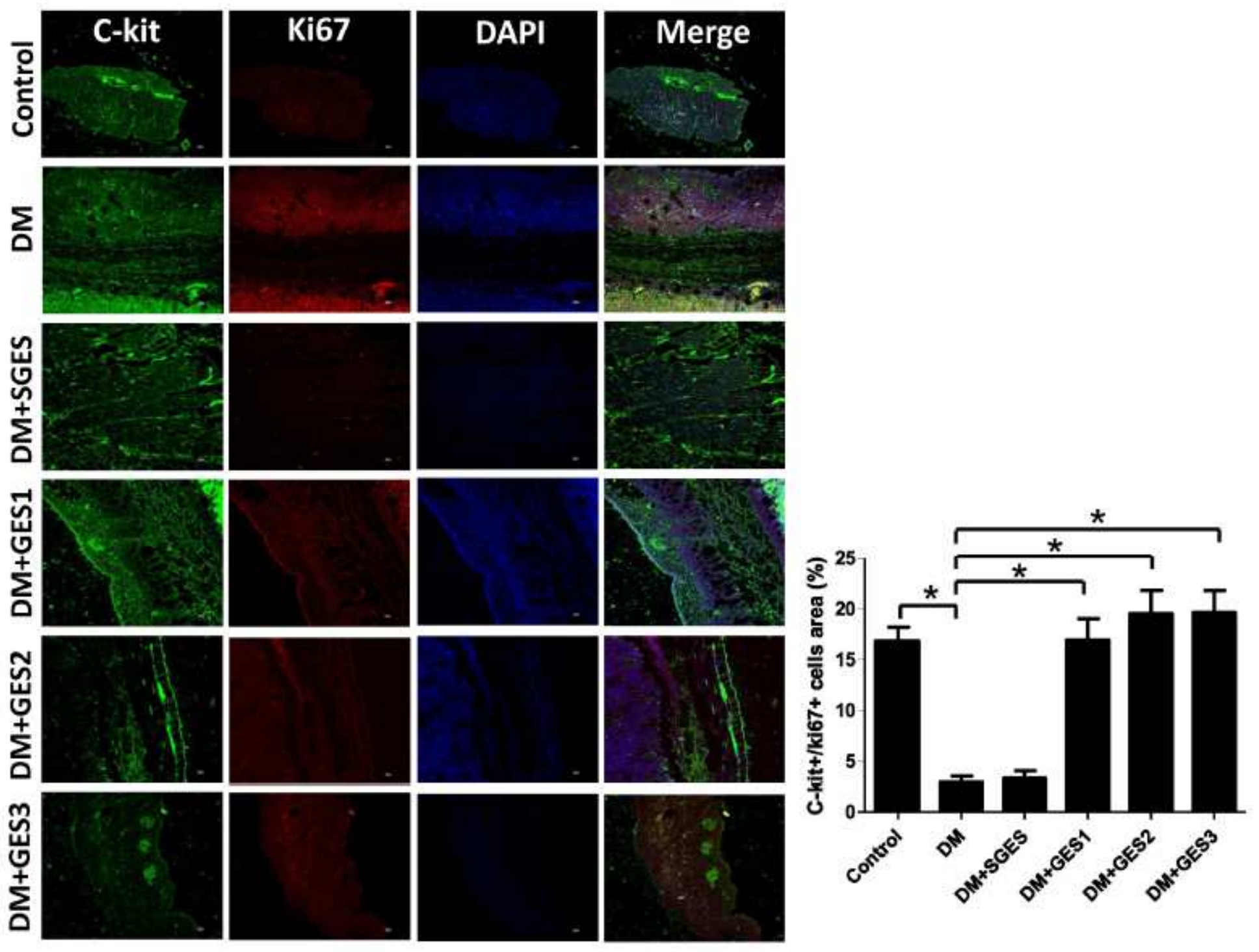

Figure 1

Representative images of immunofluorescent staining 500 for proliferated ICC labeled with c-kit (green), Ki67 (red) and DAPI (blue) in different groups. Lots of c-kit+ cells were located in the muscle and myenteric layer with dispersed Ki67+ nucleuses in the control group. An obviously decreased amount of c-kit+ cells and rarely Ki67+ nucleuses were identified in the gastric antrum of the DM and DM+SGES group. In the DM+GES groups, numerous c-kit+ cells collocated with Ki67+ nucleuses were found. Quantification of numbers of c-kit/ki67+ cells in different groups. ${ }^{*} \mathrm{P}<0.05$ compared with the $\mathrm{DM}$ group. 

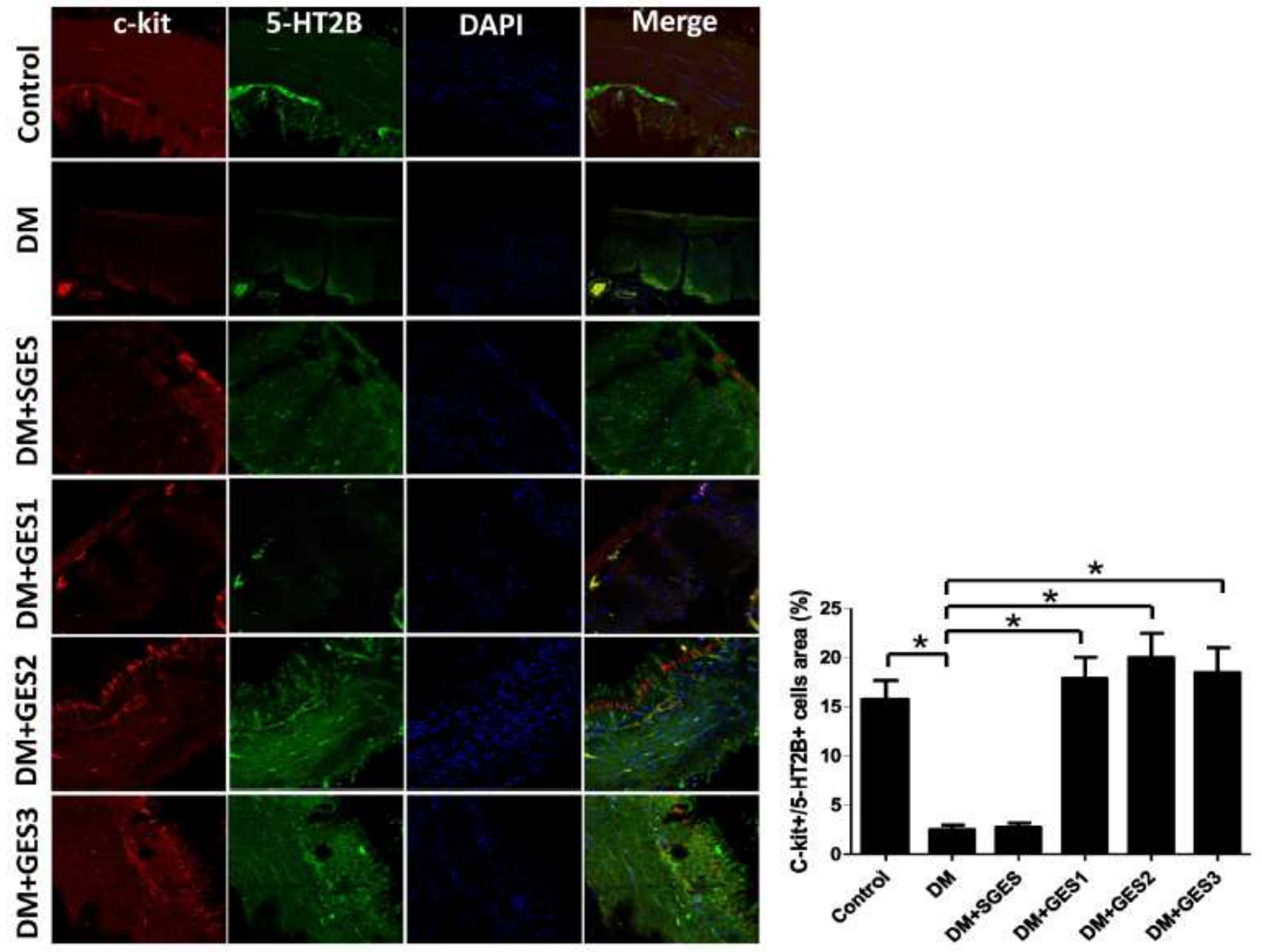

Figure 2

Confocal micrographs of 5-HT2B in the gastric antrum of different groups. The collocation of c-kit+ cells and $5-\mathrm{HT} 2 \mathrm{~B}+$ cells was in the muscle and myenteric layer in the normal rats. In the DM and DM+SGES group, the immune staining of c-kit+ and 5-HT2B+ cells were markedly reduced. 5-HT2B+/c-kit+ cells were observed to distribute in the intramuscular and myenteric layer. Scale bars $=20 \mathrm{~mm}$. Quantification of numbers of $5-\mathrm{HT} 2 \mathrm{~B}+/ \mathrm{c}-\mathrm{kit}+$ cells in different groups. ${ }^{*} \mathrm{P}<0.05$ compared with the $\mathrm{DM}$ group. 
A

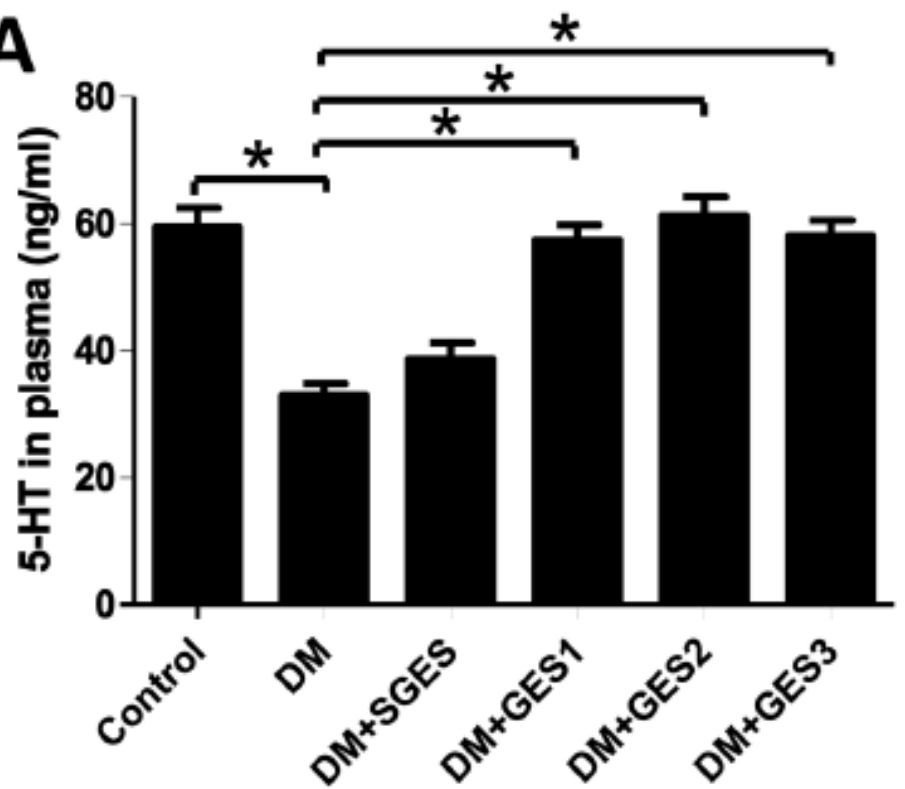

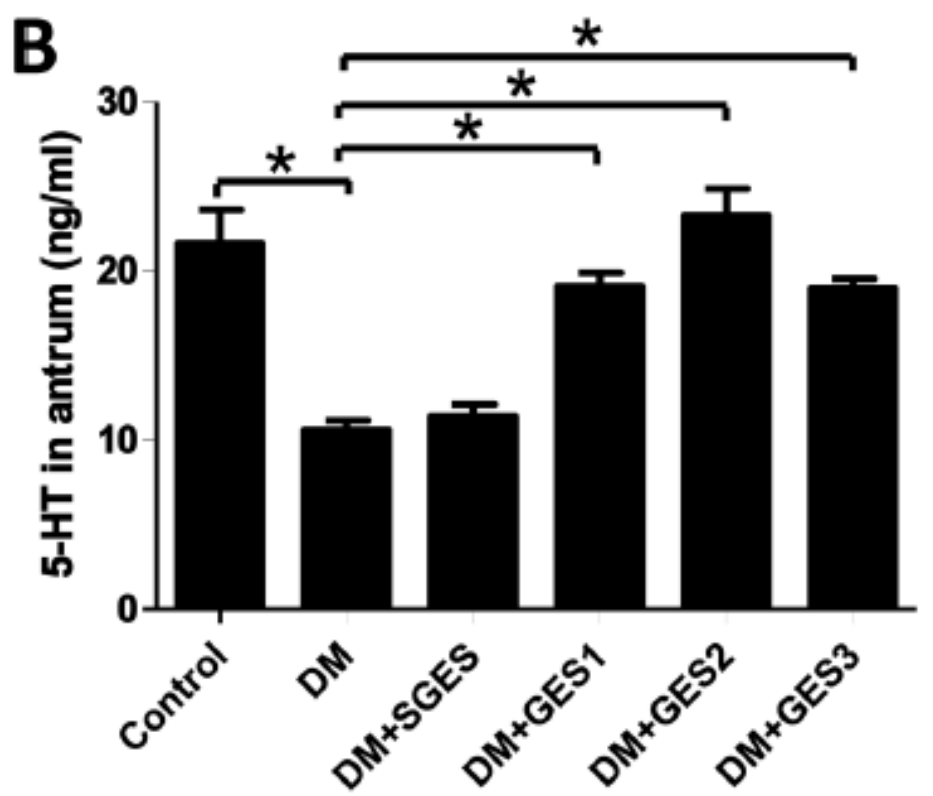

Figure 3

The plasma and tissue level of 5-HT in each group. Both the plasma and tissue 5-HT level in the DM group were significantly reduced compared the control group. However, the plasma and tissue IGF-1 level in the $D M+G E S$ groups. ${ }^{*}<<0.05$ compared with the DM group. 

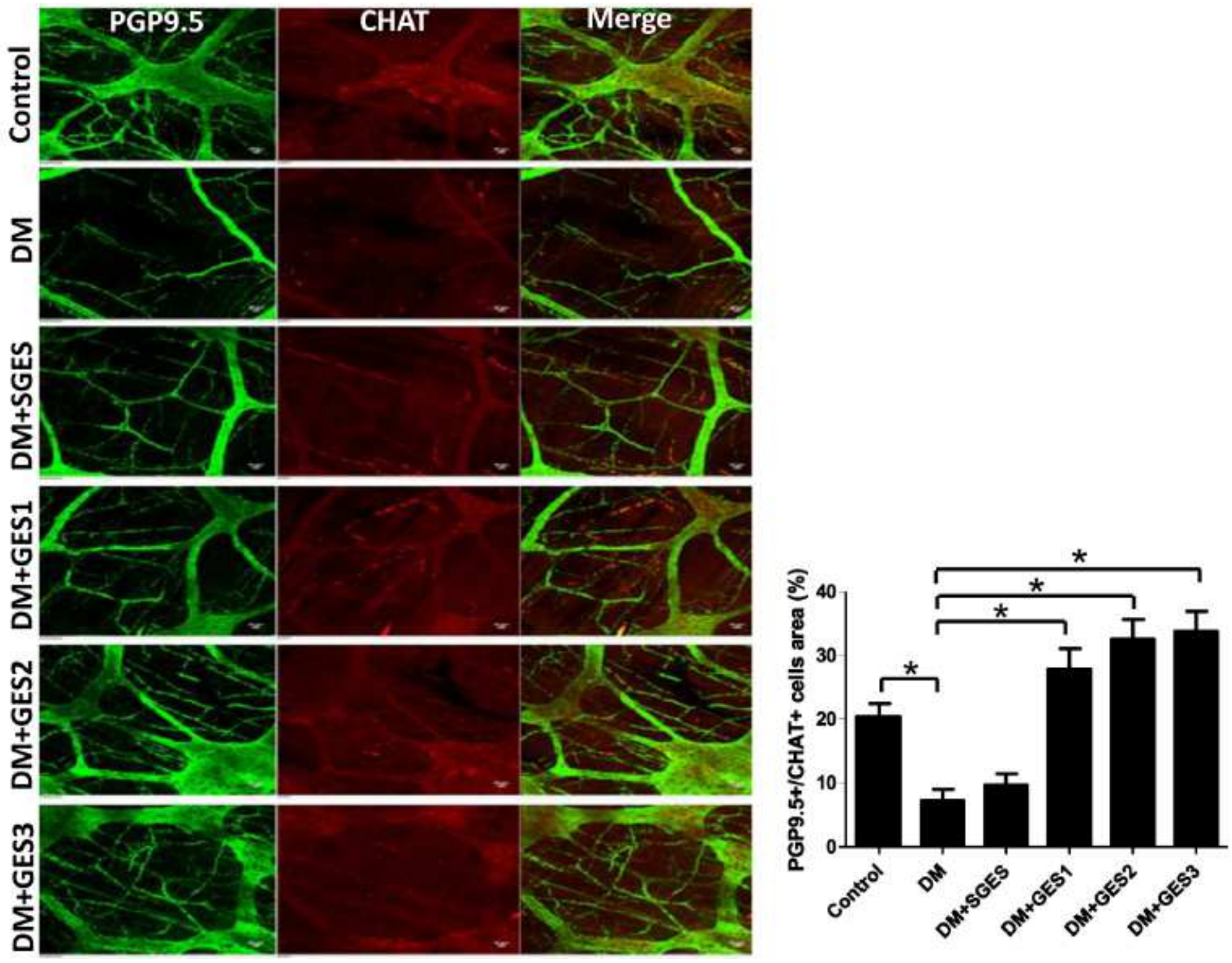

Figure 4

Confocal micrographs of cholinergic neurons labeled with PGP9.5 (red) and nNOS (green) in the myenteric layer. In the control group, a large amount of PGP9.5+/nNOS+ neurons were noted in the ganglia. Visual reductions of PGP9.5+/nNOS+ neurons were present in the DM and DM+SGES group. A remarkably increased PGP9.5+/nNOS+ neurons were showed in the DM+GES groups. Quantification of numbers of PGP9.5+/nNOS+ neurons in different 527 groups. * $\mathrm{P}<0.05$ compared with the DM group. 

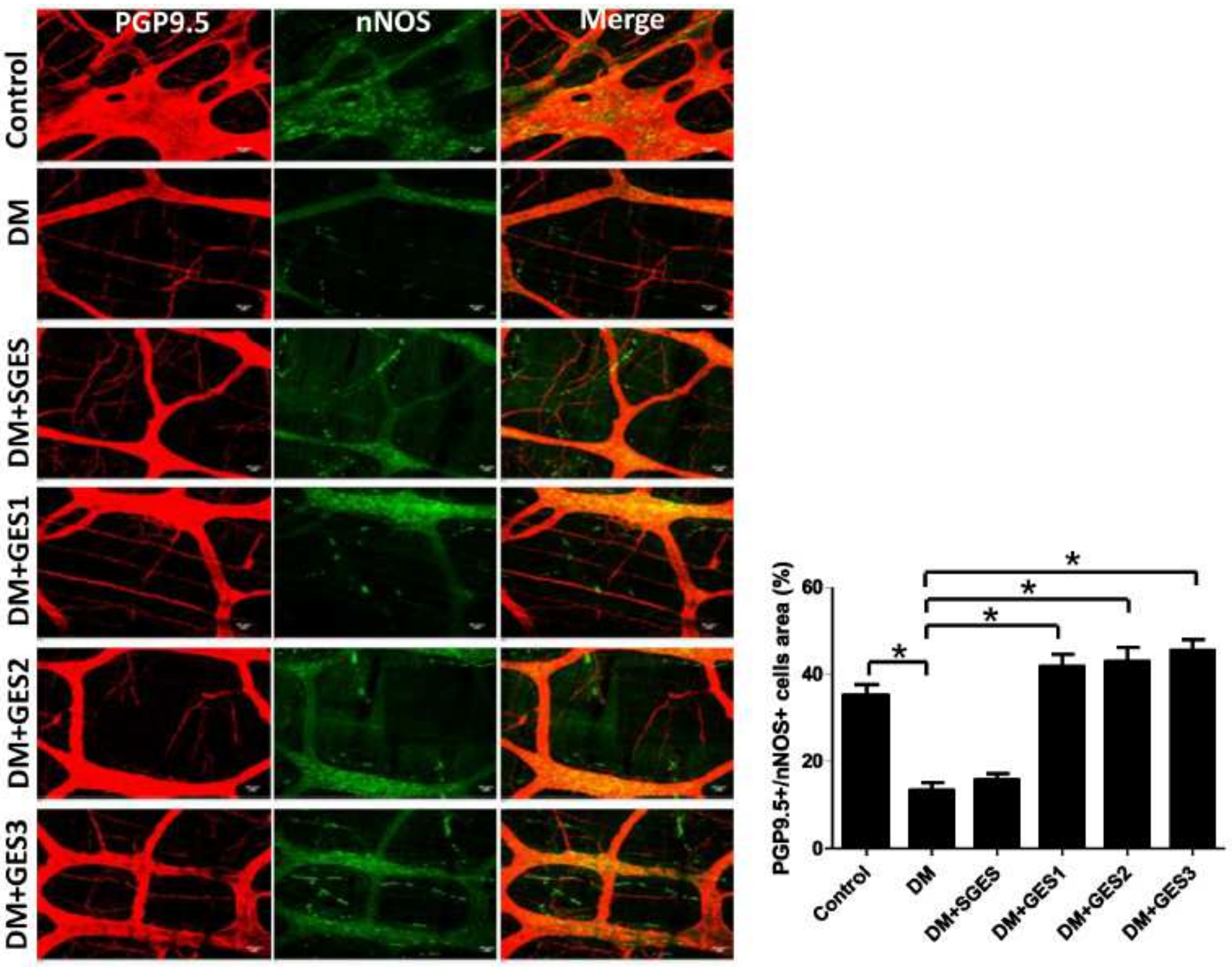

Figure 5

Confocal micrographs of cholinergic neurons labeled with PGP9.5 (green) and CHAT (red) in the myenteric layer. In the control group, plenty of CHAT staining neurons were collocated with PGP9.5 staining neurons, but a few of PGP9.5+/ CHAT+ neurons were found in the ganglia of the DM and $D M+S G E S$ group. In the DM+GES group, numerous PGP9.5+/CHAT+ neurons were observed in the myenteric layer. Quantification of numbers of PGP9.5+/CHAT+ neurons in different groups. ${ }^{*} \mathrm{P}<0.05$ compared with the DM group. 


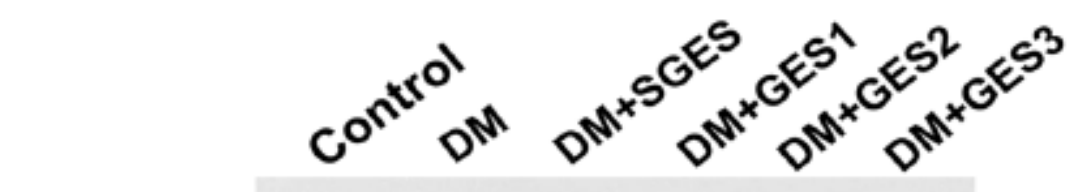

GDNF

GAPDH

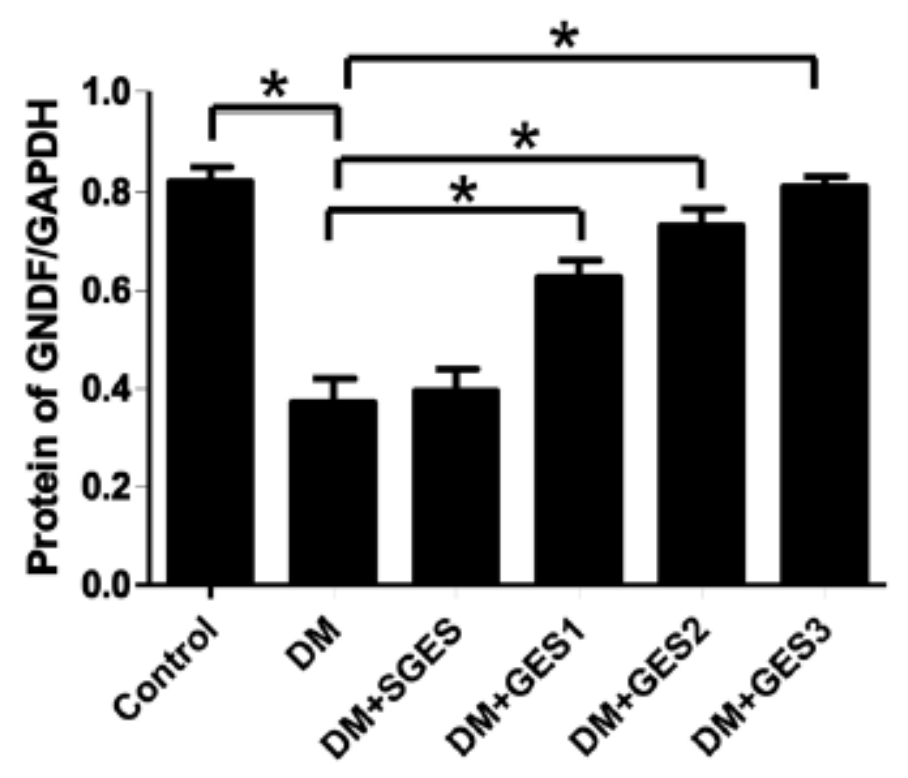

Figure 6

Expression of GNDF protein in the gastric antrum. Compared with the control group, the expression of GNDF protein was obviously decreased in the DM group. There was no significant change of GNDF protein between the DM and DM+SGES group. The expressions of GNDF protein in the DM+GES groups were improved in the $D M+G E S$ groups. ${ }^{*} P<0.05$ compared with the DM group.

\section{Supplementary Files}

This is a list of supplementary files associated with this preprint. Click to download.

- GAPDH.pdf

- GDNF.pdf 